\title{
Alles eine Frage der Logik?! Erkenntnisse einer Mixed-Method-Studie zur Pkw-Nutzung in Berlin
}

\author{
Laura Gebhardt and Rebekka Oostendorp \\ German Aerospace Center (DLR), Institute of Transport Research, \\ Rudower Chaussee 7, 12489 Berlin, Germany \\ Correspondence: Laura Gebhardt (laura.gebhardt@dlr.de)
}

Received: 29 June 2020 - Revised: 22 December 2020 - Accepted: 4 March 2021 - Published: 7 April 2021

\begin{abstract}
Kurzfassung. This paper is an empirical mixed-method study on car use in an urban context. It explores the questions: What mobility practices do people in the city display? What role does car use play in this context? What is the guiding, underlying logic behind personal car use? The findings help to understand mobility practices and their underlying logic. The central component is a user typology based on a quantitative survey and qualitative interviews. The study aims to present an empirical description of mobility practices and the guiding logic of different mobility types in Berlin. The findings offer starting points for user-specific measures to encourage people to use new mobility concepts instead of their personal car.
\end{abstract}

\section{Einleitung}

Berlin-Mitte, Montagmorgen, 11.00 Uhr: Peter Mönch (51 J.) ist nach einem Kundentermin am Morgen in BerlinMitte in Eile, um pünktlich zu einer Veranstaltung am Stadtrand zu kommen. Er läuft hastig zu seinem Wagen, für den er mit Müh und Not einen Parkplatz gefunden hat...

Berliner Stadtrand, Samstagabend, 19 Uhr: Die Witwe Paula Schmidt (70 J.) lebt in einem ruhigen Wohngebiet am Stadtrand. An diesem Abend ist ein Theaterbesuch in der Berliner Innenstadt geplant. Im Alltag erledigt sie ihre Einkäufe etc. gerne mit dem eigenen Pkw, die Fahrt mit dem Auto in der Innenstadt und die damit verbundene Parkplatzsuche findet sie jedoch grauenvoll. Daher hat sie sich entschieden, ihr Auto an der nächsten S-Bahn-Station zu parken und dann mit der S-Bahn stressfrei in die Innenstadt zu fahren...

Peter und Paula sind prototypische StellvertreterInnen für viele Menschen, die tagtäglich in der Stadt unterwegs sind. Sie nutzen - wenn auch auf unterschiedliche Art und aus unterschiedlichen Gründen - das (eigene) Auto. Und damit sind sie nicht allein: Nach wie vor ist das Auto - trotz zunehmender Mobilitätsalternativen in Städten (Lanzendorf und Hebsaker, 2017) - das meistgenutzte Verkehrsmittel (BMVI, 2017).

Angesichts dessen und der damit einhergehenden Belastung für Mensch und Umwelt gewinnt die Frage nach neu- en Mobilitätskonzepten (Docherty et al., 2017) als Alternativen zum motorisierten Individualverkehr (MIV) an Bedeutung (WBGU, 2011). In dieser stark technologiegetriebenen Diskussion sowie in der klassischen Verkehrsforschung wird Mobilität meist aus einer technischen oder organisatorischen Perspektive betrachtet. Das führt dazu, dass Mobilität von außen, sozusagen mit dem Blick der Forschenden, häufig quantitativ erfasst wird. Im Vergleich dazu sind Forschungen deutlich unterrepräsentiert, die aus einer Innenperspektive heraus die den Mobilitätspraktiken zugrunde liegenden Faktoren (interpretativ-verstehend) betrachten. NutzerInnenorientierte, subjektbezogene Studien dieser Art, die meist eher aus dem Feld der sozialwissenschaftlichen Mobilitätsals der Verkehrsforschung ${ }^{1}$ stammen, sind zumeist durch eine stark methodologisch motivierte Forschungshaltung geprägt, Probleme der Planungspraxis interessieren dabei kaum (Wilde und Klinger, 2017). In der Folge ergibt sich laut Wilde (2014: 372) ,ein Missverhältnis: Die einen schlagen Lösungen für die Planungspraxis vor, verstehen aber wenig von der Lebenspraxis der Menschen, die anderen forschen über die Lebenspraxis und versuchen, den Alltag und die Perspektive der Menschen zu entschlüsseln, überführen ihre Einblicke allerdings kaum in Erkenntnisse für die Planungspraxis“.

\footnotetext{
${ }^{1}$ Für eine Betrachtung der Abgrenzung zwischen Mobilitätsforschung und Verkehrsforschung siehe Wilde und Klinger (2017).
} 
An dieser Lücke setzt dieser Beitrag an, indem die Mobilitätspraktiken und die damit verbundenen Logiken unterschiedlicher Pkw-NutzerInnen genauer betrachtet werden. Dafür wurden mit einem Mixed-Methods-Ansatz quantitative und qualitative empirische Daten in Berlin erhoben und darauf aufbauend Mobilitätstypen identifiziert. Die Stadt Berlin dient hier als Fallbeispiel. Der Schwerpunkt des Beitrags liegt auf der Darstellung der empirischen Ergebnisse. Die folgenden Forschungsfragen leiten dabei die inhaltliche Diskussion:

- Welche Mobilitätspraktiken zeigen Menschen in der Stadt?

- Welche Rolle nimmt dabei die Pkw-Nutzung ein?

- Welche Logiken sind mit automobilen Praktiken verknüpft?

Mobilitätspraxis wird hier in Anlehnung an Wilde (2014) als alltägliches, praktisches Tun eingebettet in soziale Strukturen verstanden. Mobilität soll dadurch ,über den Akt der Raumüberwindung hinaus gehend - als sozial und kulturell konstituierte, reproduzierte Lebenspraxis aufgefasst" werden (ebd.: 160). Beim Begriff „Logiken“ geht es nicht (allein) um Rationalitäten, sondern um sämtliche intentionale wie nicht-intentionale, implizite Faktoren, die letztendlich zur Ausführung einer Praxis führen (vgl. Kapitel 2, Reckwitz, 2003: 290). Mit Berücksichtigung unterschiedlicher Mobilitätspraktiken kann der Forderung nach einer stärkeren Orientierung der Forschung an der Lebenspraxis der Menschen (z.B. Manderscheid, 2019: 179-180) begegnet werden.

Zur Beantwortung der Forschungsfragen wird eine empirisch begründete Beschreibung von unterschiedlichen Mobilitätstypen sowie deren Mobilitätspraktiken und damit verknüpfte Logiken präsentiert. Die nötige Komplexitätsreduzierung bei Typisierungen führt häufig dazu, dass eine $\mathrm{Zu}$ ordnung der Individuen zu einem dominanten Verkehrsmittel vorgenommen wird (z.B. Hunecke und Haustein, 2007). Auch die Planungspraxis betrachtet VerkehrsteilnehmerInnen häufig nur als Momentaufnahme im Straßenverkehr (AutofahrerIn, RadfahrerIn, FußgängerIn), deren Bedürfnisse es zu adressieren gilt. Allerdings sind die NutzerInnen in ihrem Alltag häufig nicht nur auf ein Verkehrsmittel fokussiert und ihre Bedürfnisse entsprechend vielfältiger. Multi- und intermodale Verkehrsmittelnutzung, also die Nutzung mehrerer unterschiedlicher Verkehrsmittel im Verlauf einer Woche bzw. eines Weges (Chlond, 2013), gehört insbesondere in Großstädten immer mehr zur Lebenspraxis der Menschen und wird daher in dieser Studie bei der Typisierung explizit berücksichtigt.

Kapitel 2 gibt einen kurzen Überblick zum Stand der Forschung bezogen auf Mobilitätspraktiken und -logiken und beschreibt die theoretische Brille der Forscherinnen bei dieser Studie. Auf dieser Basis beschreibt Kapitel 3 das methodische Vorgehen. Kapitel 4 präsentiert fünf Mobilitätstypen, die auf Grundlage des empirischen Materials mit einem Fokus auf automobile Mobilitätspraktiken und damit verbundene Logiken identifiziert und untersucht wurden. In Kapitel 5 werden die Erkenntnisse diskutiert und davon ausgehend Anknüpfungspunkte der Planungspraxis für nutzerspezifische Maßnahmen herausgestellt. In Kapitel 6 wird ein kurzes Fazit gezogen sowie weiterführender Forschungsbedarf benannt.

\section{Mobilitätspraktiken und -logiken als Elemente sozial-geographischer Mobilitätsforschung}

Obwohl sich die klassischen Determinanten der Verkehrsnachfrage (z.B. sozio-demographische Merkmale) für manche Forschende nach wie vor als die wichtigsten Erklärungsfaktoren des Mobilitätsverhaltens ${ }^{2}$ erweisen (Scheiner, 2007: 704), ist „die Verkehrsnachfrage noch immer nicht ,gut erklärbar' [...], sondern [unterliegt] einem erheblichen Eigensinn der Verkehrsnachfrager“. Es wird angenommen, dass „Verkehrshandeln möglicherweise von ganz anderen Parametern, Rationalitäten und Entscheidungslogiken bestimmt ist als denjenigen, die in der Forschung untersucht werden“" (ebd.). Die bisher dominierenden Ansätze aus klassischer Verkehrswissenschaft und Planungspraxis beobachten und erklären Mobilität meist durch isolierte, rational gefällte Entscheidungen und aus einer Außenperspektive. Die Verknüpfung von realisiertem Verkehrsverhalten mit den individuellen Lebenskontexten sowie den Mobilitätspraktiken zugrunde liegenden Logiken der NutzerInnen bleibt dabei meist unberücksichtigt.

Jüngere, sozialwissenschaftlich ausgerichtete Arbeiten der Mobilitätsforschung schenken diesen komplexen Wirkungszusammenhängen, die intentionale wie nicht-intentionale Faktoren einbeziehen, mehr Beachtung. Mobilität wird hier nicht nur als Raumüberwindung nach rationalen Entscheidungsmustern, sondern als „ein soziales Phänomen“ - als „,sozial und kulturell konstituierte Bewegung des Akteurs“ (Wilde, 2014: 36) verstanden (vgl. z.B. Hannam et al., 2006; Urry, 2007).

Bei einigen handlungstheoretischen Arbeiten wird - als bewusster Gegensatz zu behavioristischen, nur an Kennzahlen orientierten Ansätzen - der Fokus auf die Motive und Motivationen der Handelnden gesetzt (Scheiner, 2014: 149; De Vos et al., 2016; Segert, 2009). Ziel ist es, das Handeln von Individuen zu verstehen, oft als Ergebnis rational oder normativ geformter Entscheidungen. Im Anschluss

\footnotetext{
${ }^{2}$ Wenn in diesem Beitrag von „Mobilitätsverhalten“ gesprochen wird, ist das klassische Konzept quantitativ gemessener Bewegung einer Person zwischen erdräumlichen Positionen (vgl. Schopf, 2001: 5) gemeint. Ausgeklammert sind dabei noch die diesen Bewegungen zugrunde liegenden materiellen, sozialen und psychologischen Bedingungen. Diese bezieht ein komplexeres, soziotechnisches Verständnis von Mobilität ein, das sich im Begriff der „Mobilitätspraktiken“ äußert.
} 
an diese handlungstheoretische Mobilitätsforschung etabliert sich derzeit zudem eine neuere, praxistheoretische Perspektive auf Mobilität. Die Praxistheorien betrachten den Akt des Handelns und das dafür erforderliche praktische Wissen, also mehr das knowing how anstatt knowing that. Die theoretischen Grundlagen stammen aus der Soziologie (vor allem Giddens, 1984; Schatzki, 1996; Reckwitz, 2003), wurden jedoch jüngst im Feld der Mobilitätsforschung aufgegriffen (Shove et al., 2012; Wilde, 2013; Manderscheid, 2019). Der Mehrwert der Praxisperspektive auch für das Thema Mobilität liegt unter anderem in der Offenlegung von implizitem Wissen und sozio-technischen Konstellationen (Scheiner, 2014). Praxeologischen Arbeiten gelingt es, die Lücke zwischen reinem Strukturalismus auf der einen und Subjektorientierung auf der anderen Seite zu schließen. Beispielsweise versucht Wilde (2014) mit seiner Perspektive einer sozialgeographischen Mobilitätsforschung auf Alltagspraktiken älterer Menschen im ländlichen Raum das Spannungsverhältnis zwischen (routinisiertem) Verhalten, Strukturen und ,,anderen Rationalitäten“ mithilfe der Praxistheorien $\mathrm{zu}$ verstehen. Er schafft es dabei, die Innenperspektive mit den Konzepten der Praxistheorie zu verknüpfen. Ähnlich gehen Le Bris (2015), die Mobilitätspraktiken von PedelecBesitzerInnen betrachtet oder Wörmer (2016), der Praktiken analysiert, mit denen Menschen versuchen, ihr Privatleben mit den berufsbedingten Mobilitätsanforderungen zu vereinbaren, vor. Diese Arbeiten unterscheiden sich von praxistheoretischen Arbeiten, die eher an der strukturellen Formierung von Praktiken interessiert sind als an der Perspektive der Praktizierenden selbst (siehe z.B. Schatzki, 2012; Manderscheid, 2019).

Die praxeologische Brille erlaubt es, über die sprachliche Artikulation hinaus die mit menschlichem Tun einhergehenden Verkörperungen, Materialisierungen, impliziten Wissensbestände und Raumaneignungen zu erfassen. Die Praktiken werden einerseits durch die kognitiven und materiellen Strukturen geprägt - andererseits werden Praktiken und die sie verstetigenden Strukturen durch die Handelnden erst hervorgebracht und sind grundsätzlich veränderlich (Reckwitz, 2003: 296). Auch aus der eher sozial-psychologischen Forschung gibt es vergleichbare Überlegungen, zum Beispiel das Konzept der body-mind-world assemblage von Venn (2010), das von Schwanen et al. (2012: 526-527) aufgegriffen wurde, womit routinisiertes Handeln in den materiellen und sozialen Kontext eingebettet werden soll. Die grundsätzliche Zielgerichtetheit des menschlichen Handelns wird von einigen PraxistheoretikerInnen dennoch vorausgesetzt, etwa von Giddens (1984). Auch bleibt bei ihm das Potenzial eines Subjekts erhalten, strukturell reproduzierte Praktiken zu reflektieren, zu ersetzen oder anzupassen.

Angelehnt daran sollen hier Mobilitätspraktiken ebenfalls sowohl strukturell geprägt als auch durch intentionales Handeln veränderlich verstanden werden. Im Gegensatz zu manchen Praxistheorien hat hier auch das Subjekt und dessen Perspektive eine zentrale Bedeutung - in Anlehnung an die oben genannten Ansätze von Wilde (2014) und Le Bris (2015).

Von Reckwitz‘ Grundannahmen ausgehend entwirft Wilde ein Modell der Mobilitätspraxis, das Materialität, Wissen und Routinen als Grundelemente sozialer Praktiken einbezieht (Wilde, 2014). Die Materialität umfasst die Orte, Strecken, Werkzeuge und Verkehrsmittel, die zur Verwirklichung einer Praxis gehören, sowie den Körper der Praktizierenden selbst. Wissen setzt sich zusammen aus motivational-emotionalem Wissen (Motiven, Gefühlen und Stimmungen), interpretativem Verstehen (z.B. Symboliken bestimmter Orte oder Verkehrsmittel) und methodischem Wissen, also den Kompetenzen in der Planung und Ausführung bestimmter Mobilitätspraktiken. Routinen sind hier, ,gewohnheitsmäßige Handlungsabfolgen [...] in einem alltäglichen Strom sozialer Praxis“ (Wilde, 2014: 167). Dieses Modell der Grundelemente von Mobilitätspraktiken wird im weiteren Verlauf als Ausgangspunkt für die Analyse der empirischen Ergebnisse genutzt.

Neben diesen Grundelementen sozialer Praktiken soll hier außerdem das Konzept der ,,impliziten“ bzw. ,informellen“ Logik" von Praktiken aufgegriffen werden (Reckwitz, 2003: 290). Die ,implizite Logik“ einer Praktik beschreibt eine komplexe Verbindung von Intentionalität und Motivation, Routinen und Know-How (Reckwitz, 2003). Das Soziale lasse sich nur begreifen, wenn man seine Materialität und seine implizite, nicht-rationalistische Logik nachvollziehe (ebd.: 290). Schatzki (2012: 1-2) umschreibt dieses Konzept als nonpropositional ability, die Eigenschaft einer Person, die soziale, psychologische und körperliche Elemente in einem Moment zusammenbringt, um eine bestimmte Praxis zu vollführen. Giddens spricht von einem practical consciousness, das alle sozialen, materiellen und psychologischen Wissenskomponenten umfasst, die im Moment des Handelns zur Ausführung einer bestimmten, routinisierten Praxis führen (Giddens, 1984: 6-7, 167).

Anlehnend daran wird hier von einer Logik von Mobilitätspraktiken gesprochen, die - ähnlich wie Reckwitz' implizite Logik - nicht primär rationalistisch zu verstehen ist. Ähnlich wie bei Giddens' practical consciousness sollen dabei alle Elemente inbegriffen werden, die die Ausführung einer bestimmten Praktik erklären können. Der Begriff soll also als Rahmen dienen, um alle möglichen Elemente - routinisierte wie intentionale -, die tatsächlich zur Ausführung der Praxis geführt haben, zu identifizieren und zusammenzufassen.

\section{Methodik}

Ausgangspunkt für die vorliegende Mixed-Methods-Studie ist die Annahme, dass Akteure Mobilität unter den Bedingungen ihres Lebensalltags produzieren und reproduzieren (Hannam et al., 2006; Dangschat und Segert, 2011). Unser sequentieller Mixed-Methods-Ansatz hat dabei die Identifi- 
zierung und das Verstehen unterschiedlicher Mobilitätstypen und deren Mobilitätspraktiken zum Ziel. Damit verbunden ist die Annahme, dass bestimmte Mobilitätspraktiken durch bestimmte Gruppen getragen werden, die durch Lebenssituation, strukturelle Faktoren sowie soziodemographische Faktoren charakterisiert sind (Shove et al., 2012). Eine gruppenspezifische Betrachtung kann helfen, bedarfsgerechte Maßnahmen zu entwickeln, die unter Umständen zu Verhaltensänderungen führen können (Dangschat, 2017).

Segmentierungsansätze sind ein etabliertes methodisches Mittel zur Analyse und Strukturierung von heterogenen Gruppen bzw. deren Praktiken und helfen, Komplexität zu reduzieren (Anable, 2005; Bartz, 2015; Manderscheid, 2019). Disziplinen, die mit Segmentierungsansätzen arbeiten, umfassen die Psychologie (z.B. Hunecke und Haustein, 2007; Hunecke, 2015), die Soziologie (z.B. Bolte, 2000; Jensen, 2009) und auch die Verkehrswissenschaften (Haustein und Nielson, 2016; Wittwer, 2014). Meistens werden die Typologien mittels quantitativer Daten und Clusteranalysen erarbeitet.

Die Arbeit mit Mobilitätstypen weist Parallelitäten zur aus der Produktentwicklung stammenden „Persona-Methode“ (Cooper, 2004) auf, da hier ebenfalls mit prototypischen NutzerInnen gearbeitet wird. Im Gegensatz zu den dort erstellten Personae hat der vorliegende Ansatz nicht eine Konsumentin bzw. einen Konsumenten als Verständnisgrundlage, sondern bestimmte Praktiken in ihren jeweiligen Kontexten. Wissenschaftlich erarbeitete Typologien sind von Personae außerdem abzugrenzen, da letztere meist durch design-thinkingProzesse ohne wissenschaftlich fundierte empirische Basis beschrieben und primär für Produktentwicklung genutzt werden (Chapman und Milham, 2006).

Unser Mixed-Methods-Ansatz geht über die reine Identifikation von Typen durch quantitative Daten hinaus, indem neben der quantitativen Erhebung und Identifizierung von Typen diese und deren Logiken im qualitativen Teil der Studie „verstanden“ und näher beschrieben werden. Stärken quantitativer sowie qualitativer Methoden bzw. Daten werden somit im Sinne von Mixed-Methods aufsummiert (Johnson et al., 2007; Kuckartz, 2016). Ein weiterer Mehrwert eines Mixed-Methods-Ansatzes liegt in der Robustheit der Ergebnisse (ebd.). Die Methoden-Triangulation hilft, dass sich verschieden erhobene Daten gegenseitig stützen und validieren oder blinde Flecken bzw. Analysefehler erkannt werden (Johnson und Onwuegbuzie, 2004).

Abbildung 1 zeigt das methodische Vorgehen des sequenziellen Mixed-Methods-Ansatzes. Der Schwerpunkt dieses Beitrags liegt auf dem qualitativen Teil der Studie, da die Interviews tiefergehende Informationen hinsichtlich der Forschungsfragen dieses Beitrags geben. Die vorhergehenden quantitativen Analyseschritte sind ausführlich in Oostendorp et al. (2019) dokumentiert.

\subsection{Identifizierung von Mobilitätstypen}

Die Grundlage für die Mobilitätstypen bildet eine im Jahr 2016 durchgeführte quantitative Befragung in Berlin zum aktuellen Mobilitätsverhalten mit 1098 Personen (vgl. Oostendorp und Gebhardt, 2018). Die TeilnehmerInnen entstammen einer geschichteten Zufallsstichprobe aus dem Einwohnermelderegister. Die Befragung ist auf der Individualebene konzipiert, enthält jedoch auch Informationen zum Haushaltskontext des Individuums, wie beispielsweise der Haushaltszusammensetzung oder der Anzahl der im Haushalt vorhandenen Pkw. Der Fragebogen erfasste unter anderem in detaillierter Weise das Mobilitätsverhalten der Befragten, differenziert nach Verkehrsmittelnutzung und Wegezwecken. Die abgefragten Variablen zur Häufigkeit unimodaler und intermodaler Verkehrsmittelnutzung für unterschiedliche Wegezwecke dienten als Einflussvariablen für eine kombinierte Faktor- und Clusteranalyse. Die daraus resultierenden Cluster werden entsprechend der Einflussvariablen nicht allein durch ein Verkehrsmittel bestimmt, sondern zeigen das vielfältige Mobilitätsverhalten im Alltag der Befragten. Die multi- und intermodalen Verhaltensweisen der BefragungsteilnehmerInnen sind demnach von zentraler Bedeutung für die quantitative Segmentierung und infolgedessen für die Unterscheidung der Mobilitätstypen. Die Cluster bilden schließlich die Basis für die Mobilitätstypen, die anschließend mit weiteren Informationen aus der Befragung zu sozio-demographischen Merkmalen und verfügbaren Mobilitätsressourcen charakterisiert wurden. Aufgrund dieses Vorgehens grenzen sich die Mobilitätstypen tatsächlich durch ihr Mobilitätsverhalten voneinander ab und die Typisierung ist nicht ein Ergebnis sozio-demographischer Unterschiede der Befragten.

Vor dem Hintergrund der Fragestellungen (vgl. Kapitel 1) wurden fünf Typen als Untersuchungsgruppen für die qualitative Erhebung ausgewählt, die im Ergebnisteil (vgl. Kapitel 4) dargestellt werden. Bei der Auswahl der Typen wurde darauf geachtet, dass sie unterschiedliches Mobilitätsverhalten aufweisen, um verschiedene Perspektiven auf PkwNutzung aus den Interviews abzubilden. Für eine Übersicht aller Mobilitätstypen sowie zur methodischen Vorgehensweise bei der Typenbildung siehe Oostendorp et al. (2019).

\subsection{Exploration der Nutzerperspektive mittels qualitativer Interviews mit visuellen Elementen}

Es wurden 22 qualitative Tiefeninterviews mit StellvertreterInnen der ausgewählten Mobilitätstypen in Berlin im Jahr 2018 durchgeführt. Die InterviewpartnerInnen konnten gezielt aus den TeilnehmerInnen der quantitativen Befragung ausgewählt werden. Sie stehen für den jeweiligen Mobilitätstyp, decken in der Summe aber auch eine gewisse Bandbreite unterschiedlicher Personen- und Haushaltsmerkmale ab.

Die Interviews wurden durch die „Nadelmethode“ (Rohrauer, 2014) sowie „Fotogeleitete Hervorlockung“ (Har- 


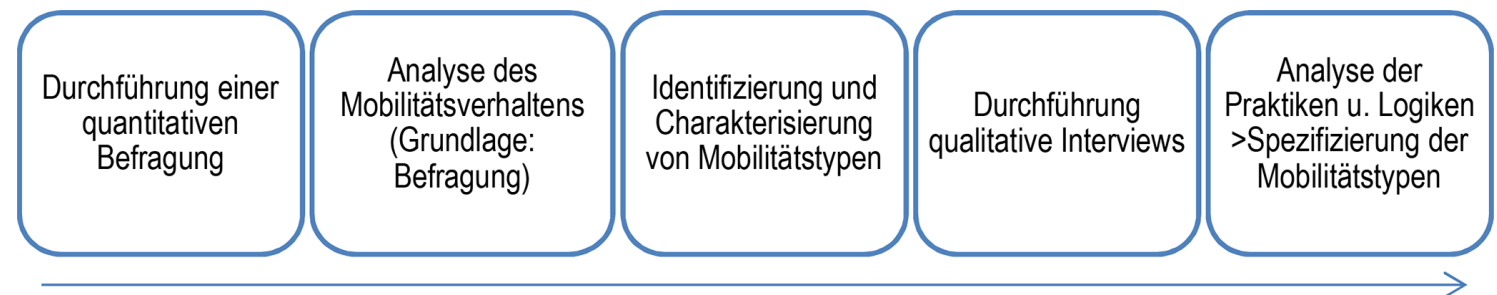

Abb. 1. Methodisches Vorgehen.

per, 2009) angereichert. Bei der Nadelmethode handelt es sich um eine Methode der Sozialraumanalyse, deren Ziel es ist, die persönlichen Lebensräume der Befragten und deren subjektive Relevanz zu rekonstruieren, indem sie relevante Punkte ihres Alltags und ihre Wege mit Hilfe von Nadeln auf einer vorgelegten Karte markieren (Rohrauer, 2014). In dieser Studie wurde die Grundidee dieser Methode genutzt und mit Aspekten der Methode der „narrativen Landkarte“ (Behnken und Zinnecker, 2010) ergänzt, jedoch in digitaler Form angewandt. Die befragten Personen wurden aufgefordert, ihre alltäglichen Wege in eine digitale Karte auf einem Tablet einzuzeichnen und dabei mündlich zu beschreiben.

Das ethnographische Verfahren der „Fotogeleiteten Hervorlockung " (Collier und Collier, 1986; Harper, 2009) dient als visueller Diskussionsstimulus im Interview und hat sich vor allem für das Verständnis der Alltagspraktiken aus der Perspektive der Befragten bewährt. So wurden die Interviewten aufgefordert, die ihnen visuell vorgelegten Mobilitätsoptionen (in Form von Fotos) zu beurteilen, deren Nutzung und Nicht-Nutzung zu bewerten, eine Auswahl zu treffen und zu begründen. Gerade Diskrepanzen zwischen der Rationalisierung der Praktiken durch die Befragten selbst und deren erfasstes Verhalten sowie ihr implizites, nicht kommuniziertes Wissen lassen sich durch den gewählten Ansatz besser identifizieren, wenngleich ein solches Vorgehen zeitintensiv ist.

Die Interviews wurden transkribiert und in Anlehnung an die Grundelemente der Theorie sozialer Praktiken - Materialität, Wissen, und Routinen (vgl. Reckwitz, 2003) - strukturiert und analysiert. Diese Kategorisierung wurde - im Sinne der „Grounded Theory“ (Charmaz, 2014; Glaser und Strauss, 1967) - durch Aspekte aus dem empirischen Material im zirkulären Auswertungsprozess kontinuierlich ergänzt.

\section{Ergebnisse}

Im Folgenden werden die fünf unterschiedlichen Mobilitätstypen, deren Mobilitätspraktiken sowie die mit den Praktiken verbundenen Logiken beschrieben. Vor dem Hintergrund der eingangs formulierten Forschungsfragen wird der Fokus auf automobilen Praktiken und den damit verbundenen Logiken liegen, um zu verstehen, in welchen Situationen Personen einen Pkw nutzen. Neben Informationen aus der quantitativen Befragung sind vor allem die Narrationen der Interviewten beim Zeichnen eines typischen Alltagsweges im Rah- men der qualitativen Interviews die Grundlage dafür. Dabei sprechen wir zum Beispiel von Peter, dem Allzweck-PkwNutzer, er ist als Stellvertreter für seine Gruppe zu verstehen. Die Inhalte und Zitate stammen von unterschiedlichen Interviewten des jeweiligen Typs.

\subsection{Beschreibung von Mobilitätstypen}

Tabelle 1 zeigt auf Grundlage der Daten aus der quantitativen Befragung eine Übersicht über die Merkmale der fünf für die qualitative Erhebung ausgewählten und für die Fragestellung in diesem Beitrag relevanten Mobilitätstypen. Die deskriptive Beschreibung der Typen dient dazu, die folgenden qualitativen Ergebnisse einordnen zu können.

Bei der Gegenüberstellung der Merkmale auf Grundlage der quantitativen Befragung wird deutlich, dass die Mobilitätstypen jeweils ein klares Profil nicht nur hinsichtlich ihres Mobilitätsverhaltens haben, sondern auch Unterschiede in den sozio-demographischen Merkmalen und der Verfügbarkeit von Mobilitätsressourcen bestehen. Die Unterschiede bei der Verkehrsmittelnutzung sind durch die bei der Clusteranalyse verwendeten Input-Variablen bedingt (vgl. Kapitel 3.1). Die multi- und intermodalen Verhaltensweisen der BefragungsteilnehmerInnen als zentraler Bestandteil der quantitativen Segmentierung zeigen sich entsprechend deutlich in der Unterschiedlichkeit der Mobilitätstypen. Gleichzeitig sind trotz unterschiedlichen Mobilitätsverhaltens auch Gemeinsamkeiten zwischen einzelnen Typen erkennbar. So weisen der Allzweck-Pkw-Nutzer und die intermodale Pkwund-ÖV-Kombiniererin beide eine hohe Pkw-Verfügbarkeit und einen geringen Anteil an ÖV-Tickets auf. Die Typen Allzweck-Pkw-Nutzer, Fahrrad-Kombinierer und situationsabhängige multimodale Nutzerin ähneln sich dagegen in ihren sozio-demographischen Merkmalen und sind alle durch einen hohen Anteil berufstätiger Personen in Familienhaushalten geprägt.

Die quantitativ identifizierten Mobilitätstypen ermöglichen es, durch ihre Charakteristika verschiedene Arten der Pkw-Nutzung bei dem im Folgenden dargestellten qualitativen Analyseschritt der Mixed-Methods-Studie zu betrachten, die in unterschiedliche Kontexte des multi- und intermodalen Mobilitätsverhaltens sowie in unterschiedliche soziodemographische und ressourcenbezogene Rahmenbedingungen der Lebenspraxis eingebettet sind. Dadurch kann ein 
Tabelle 1. Merkmale der Mobilitätstypen auf Grundlage der quantitativen Befragung.

\begin{tabular}{|c|c|c|c|}
\hline Mobilitätstyp $^{1}$ & $\begin{array}{l}\text { Mobilitätsverhalten } \\
\text { (auf Basis der Clusteranalyse; über- } \\
\text { / unterdurchschnittlich bezogen auf } \\
\text { gesamten Datensatz) }\end{array}$ & $\begin{array}{l}\text { Vorwiegende sozio- } \\
\text { demographische Merkmale }\end{array}$ & $\begin{array}{l}\text { Vorwiegende Verfügbarkeit } \\
\text { von Mobilitätsressourcen }\end{array}$ \\
\hline $\begin{array}{l}\text { Allzweck-Pkw-Nutzer } \\
\text { (unimodal) } \\
\text { (Peter) }\end{array}$ & $\begin{array}{l}\text { - hohe unimodale Pkw-Nutzung } \\
\text { zu allen Wegezwecken } \\
\text { - ergänzende Fahrradnutzung } \\
\text { - geringe Nutzung intermodaler } \\
\text { Kombinationen }\end{array}$ & $\begin{array}{l}\text { - eher männlich } \\
\text { - stärkste Altersgruppen: } \\
36-45 \text { und 46-55 Jahre } \\
\text { - hoher Anteil Berufstätiger } \\
\text { - viele Familienhaushalte } \\
\text { - leben häufig am Stadtrand }\end{array}$ & $\begin{array}{l}\text { - Pkw-Verfügbarkeit und } \\
\text { Anteil Carsharing } \\
\text {-Mitgliedschaften hoch } \\
\text { - niedriger Anteil } \\
\text { ÖV-Tickets }\end{array}$ \\
\hline $\begin{array}{l}\text { ÖV-Nutzerin } \\
\text { (Olga) }\end{array}$ & $\begin{array}{l}\text { - hohe tägliche ÖV-Nutzung } \\
\text { (uni- und intermodal) } \\
\text { - vor allem auf Arbeitswegen und } \\
\text { zu Freizeitzwecken }\end{array}$ & $\begin{array}{l}\text { - eher weiblich } \\
\text { - stärkste Altersgruppe: } \\
\text { 26-35 Jahre } \\
\text { - hoher Anteil StudentInnen } \\
\text { und SchülerInnen } \\
\text { - viele Ein-Personen- } \\
\text { und Paarhaushalte } \\
\text { - leben häufig in gut } \\
\text { angebundenen Quartieren }\end{array}$ & $\begin{array}{l}\text { - hoher Anteil ÖV-Tickets } \\
\text { - Pkw-Verfügbarkeit } \\
\text { sehr niedrig } \\
\text { - Anteil Carsharing- } \\
\text { Mitgliedschaften } \\
\text { unterdurchschnittlich }\end{array}$ \\
\hline $\begin{array}{l}\text { Intermodale Pkw- und } \\
\text { ÖV-Nutzerin } \\
\text { (Paula) }\end{array}$ & $\begin{array}{l}\text { - kombiniert häufig Pkw und ÖV, } \\
\text { vor allem zum Einkaufen und für } \\
\text { private Erledigungen } \\
\text { - außerdem hohe unimodale } \mathrm{Pkw}- \\
\text { Nutzung }\end{array}$ & $\begin{array}{l}\text { - eher weiblich } \\
\text { - stärkste Altersgruppe: } \\
\text { 66-75 Jahre } \\
\text { - Hoher Anteil RentnerInnen } \\
\text { - viele Paarhaushalte } \\
\text { - leben häufig am Stadtrand }\end{array}$ & $\begin{array}{l}\text { - Pkw-Verfügbarkeit sehr } \\
\text { hoch } \\
\text { - Nur wenige haben ein } \\
\text { ÖV-Ticket oder Carsharing- } \\
\text { Mitgliedschaft }\end{array}$ \\
\hline $\begin{array}{l}\text { Intermodale Fahrrad- } \\
\text { Kombinierer (Steffen) }\end{array}$ & $\begin{array}{l}\text { - kombiniert häufig Fahrrad } \\
\text { und ÖV } \\
\text { - intermodal zu vielen verschiede- } \\
\text { nen Zwecken, auch auf Arbeitswe- } \\
\text { gen } \\
\text { - auch unimodale Fahrradnutzung, } \\
\text { vor allem für Einkaufen, Freizeit } \\
\text { und private Erledigungen } \\
\text { - unimodale Pkw-Nutzung unter- } \\
\text { durchschnittlich }\end{array}$ & $\begin{array}{l}\text { - eher männlich } \\
\text { - stärkste Altersgruppe: } \\
\text { 36-45 Jahre } \\
\text { - hoher Anteil Berufstätiger, } \\
\text { vor allem in Vollzeit } \\
\text { - viele Familienhaushalte } \\
\text { - leben häufig in urbanen } \\
\text { Quartieren }\end{array}$ & $\begin{array}{l}\text { - Anteil ÖV-Tickets und } \\
\text { Carsharing-Mitgliedschaf- } \\
\text { ten hoch } \\
\text { - Pkw-Verfügbarkeit } \\
\text { vergleichsweise gering }\end{array}$ \\
\hline $\begin{array}{l}\text { situationsabhängige } \\
\text { multimodale Nutzerin } \\
\text { (Silvia) }\end{array}$ & $\begin{array}{l}\text { - sowohl intermodal als auch } \\
\text { unimodal unterwegs } \\
\text { - intermodale Kombinationen für } \\
\text { alle Wegezwecke überdurch- } \\
\text { schnittlich } \\
\text { - zusätzlich hohe unimodale } \\
\text { Pkw- und Fahrradnutzung }\end{array}$ & $\begin{array}{l}\text { - eher weiblich } \\
\text { - stärkste Altersgruppen: } \\
\text { 46-55 Jahre und 56-65 Jahre } \\
\text { - hoher Anteil Berufstätiger } \\
\text { - viele Familienhaushalte } \\
\text { - leben häufig am Stadtrand }\end{array}$ & $\begin{array}{l}\text { - Pkw-Verfügbarkeit und } \\
\text { Anteil ÖV-Tickets ist et- } \\
\text { wa gleich hoch auf einem } \\
\text { durchschnittlichen Niveau } \\
\text { - Anteil Carsharing- } \\
\text { Mitgliedschaften sehr } \\
\text { gering }\end{array}$ \\
\hline
\end{tabular}

${ }^{1}$ Bei den Namen der Mobilitätstypen wurde jeweils das Geschlecht gewählt, dass bei diesem Typ im Datensatz stärker vertreten ist. D.h. Peter der Allzweck-Pkw-Nutzer und Paula die ÖV-Nutzerin.

2 ÖV-Ticket meint hier den Besitz einer Zeitfahrkarte (Monats- oder Jahrestickets) für den ÖV.

breites Spektrum an verschiedenen Lebenswelten und damit verbundenen Perspektiven auf die Thematik der PkwNutzung in den Interviews berücksichtigt werden.

\subsection{Mobilitätspraktiken und -logiken unterschiedlicher Mobilitätstypen}

In Anlehnung an Reckwitz (2003) bzw. Wilde (2014) wurden die drei Haupt-Analysekategorien Materialität, Wissen und Routinen für die Analyse und Ergebnisdarstellung gewählt. Bezogen auf das hier in den Blick genommene Thema 
Pkw-Nutzung lässt sich die Kategorie Materialität anhand der Aspekte Pkw-Verfügbarkeit und deren Bedeutung für den Mobilitätstyp, Aussagen zum Transport von Personen und Gepäck sowie zu Kosten analysieren. Außerdem sind die beschriebenen Orte und Strecken dieser Kategorie zuzuordnen. Die Kategorie Wissen lässt sich in den Ergebnissen durch methodisches Wissen über die Nutzungsweise der einzelnen Verkehrsmittel, Ortskenntnisse des Straßen- und ÖVNetzes, die Nutzung von Mobilitäts-Apps und Carsharing, aber auch durch motivational-emotionales Wissen und interpretatives Verstehen (z.B. symbolische Aufladung von Verkehrsmitteln und Orten) darstellen. Vor allem die Narrationen zu den auf eine Karte gezeichneten Alltagswegen zeigen implizites Wissen der Befragten. Aussagen zu Routinen lassen sich im empirischen Material zum Beispiel durch Beschreibungen des Arbeitsweges, Aussagen zu Zeit und Flexibilität oder auch Aussagen zu häufig wiederkehrenden Stausituationen und der Parkplatzsuche finden.

\section{Peter (Allzweck-Pkw-Nutzer)}

Für den Allzweck-Pkw-Nutzer Peter ist der Pkw das optimale Verkehrsmittel, das selten in Frage gestellt wird: „,Und deswegen ist Auto eigentlich so das Standardverkehrsmittel" (A4). Obwohl Peter von manchen Aspekten der PkwNutzung, wie dem täglichen Stau in der Stadt, genervt ist, gibt es praktisch keine Abwägung zwischen unterschiedlichen Mobilitätsoptionen. Die Pkw-Nutzung ist ein fester, hochroutinierter Bestandteil des Alltags geworden. Die Materialität des Pkws wird dem Anspruch nach Flexibilität, Komfort und Privatheit gerecht. Der Pkw-Besitz wird als gesellschaftlicher Normalzustand gesehen, wie ein Interviewter zum Ausdruck bringt:,, Wir haben einen großen und einen kleinen [Pkw], wie das so üblich ist und da geht es dann bei Fernstrecke der große, der kann mehr" (A1). Als einen typischen Alltagsweg zeichnen die meisten InterviewpartnerInnen ihren Arbeitsweg. Abbildung 2 (links) zeigt beispielhaft den täglichen Weg von Peter vom Wohnort zur Schule der Tochter und dann quer durch die Stadt zur Arbeitsstätte.

Bei gutem Wetter wird nach dem Pkw am ehesten das Fahrrad diesen Anforderungen gerecht. Die Selbstbestimmtheit beider Verkehrsmittel scheint hier ein zentraler Aspekt zu sein. Sich an die Materialität eines vorgegebenen Systems, an Abfahrtzeiten und vorgegebene Routen anzupassen, wird als Einschränkung empfunden. Daher wird der ÖV auch nicht als echte Option wahrgenommen. Dabei verfügt Peter kaum über fundiertes Wissen zu potentiellen Verkehrsmittelalternativen. Stattdessen liegt eine gute Ortskenntnis des Straßennetzes (vor allem auf Routinewegen) vor.

Der Transport von Personen (insb. Kindern), Tieren und Gepäck ist ein weiterer, essentieller Grund für die PkwNutzung im Alltag. In diesem Punkt wird die Materialität der Pkw-Nutzung besonders deutlich. Das ,Alles-untereinen Hut-bringen“ eines komplexen Alltags ist vor allem für Mütter ein Grund für die Auto-Nutzung. Der Pkw er- möglicht eine flexible, individuelle Planung, mehrere Wegezwecke können kombiniert werden. Er wird als zeiteffizient und kostengünstig empfunden, wobei im Gespräch indirekt zum Ausdruck kommt, dass Staus, die tägliche Parkplatzsuche sowie versteckte Kosten diese Argumente auch entkräften könnten. Hier zeigt sich, dass die Logiken des AllzweckPkw-Nutzers nicht auf ein rein zweckrationales Abwägen reduziert werden können. Teil dessen Logik ist, dass psychologische und normative Elemente diese in der Praxis verborgenen Paradoxien verschleiern und sie dadurch subjektiv nach sozial erwünschten Maßstäben interpretiert und rationalisiert werden. Hier spielen emotional-motivationale Aspekte, wie das Empfinden des eigenen Autos als ein zweites Wohnzimmer oder als ein Rückzugsraum, eine zentrale Rolle. Im Artefakt Auto materialisiert sich sozusagen die Logik dieses Typs.

\section{Olga (ÖV-Nutzerin)}

Die ÖV-Nutzerin Olga wohnt in der Regel in einem gut angebundenen urbanen Gebiet und hat eine Auswahl an Verkehrsmitteln zur Verfügung. Die meisten Befragten dieses Typs haben kein eigenes Auto. Bei der Entscheidung gegen das Auto spielen die Kosten und die verlorene Zeit in Staus und bei der Parkplatzsuche eine entscheidende Rolle. Wenn etwas transportiert werden muss oder für Fahrten in Gebiete außerhalb der Stadt, wird gelegentlich ein Pkw genutzt. Das Fahrrad ist für diese Gruppe wiederum keine attraktive Alternative, da es zu wetterabhängig ist und weniger Komfort als der ÖV bietet. Der ÖV stellt für sie im Vergleich zu anderen Alternativen die komfortabelste, flexibelste sowie zeit- und kosteneffizienteste Mobilität dar. Häufig werden verschiedene Verkehrsmittel des ÖV genutzt. Anders als der Allzweck-Pkw-Nutzer Peter hat Olga dabei methodisches Wissen darüber, welche Verkehrsmittel wann und wo verfügbar sind. Welche Verkehrsmittel letztendlich gewählt werden, wird situations- und tagesabhängig entschieden mal ist die Zeitersparnis wichtiger, mal der Komfort. In der Abwägung der Vor- und Nachteile der Alternativen zeigt sich ihr Wissen in Form von interpretativem Verstehen. Es besteht also keine Routine bei der Nutzung eines bestimmten Verkehrsmittels, vielmehr ist die Praktik des flexiblen Agierens eine Routine. Die Entscheidung für den ÖV und gegen das Auto fällt dabei pragmatisch und nicht, weil eine grundsätzliche emotional-motivationale Präferenz besteht. Im Gegenteil scheint der ÖV allein deswegen häufig das Verkehrsmittel der Wahl zu sein, weil es für Olga in vielen Situationen weniger nachteilhafte Aspekte hat als andere Verkehrsmittel. Kein Auto zu haben oder es in der Stadt nicht zu nutzen, sondern stattdessen zwischen verschiedenen ÖV-Optionen wählen zu können, wird teils als grundsätzlich positiver $\mathrm{Zu}$ stand gesehen: „Es hat tatsächlich was mit Lebensqualität zu tun, dass man sich eben nicht stresst, sondern eben doch mit der Straßenbahn fährt. [...] in der Stadt bedeutet Auto eher Stress “(O4). Hier kommt das Wissen im Sinne des 


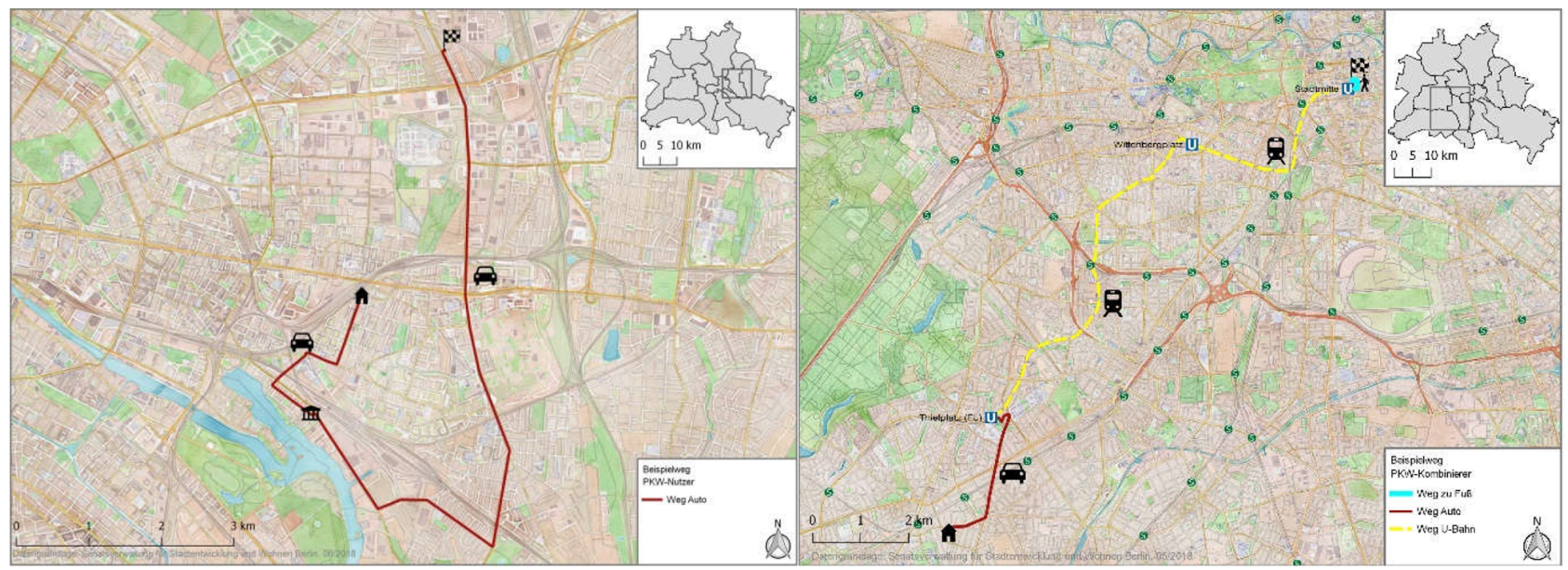

Abb. 2. Im Interview gezeichneter typischer Alltagsweg vom Allzweck-Pkw-Nutzer (links) und der Pkw-und-ÖV-Kombiniererin (rechts). Rot $=$ Pkw; gelb $=$ ÖV; hellblau $=$ zu Fuß. Kartengrundlage: FIS Broker 2019.

emotional-motivationalen Wissens (Vermeidung von Stress als Motiv) und interpretativen Verstehens (Einfluss auf persönliche Lebensqualität) des Typs noch einmal deutlich zum Ausdruck.

\section{Paula (Intermodale Pkw- und ÖV-Kombiniererin)}

Für die Pkw-und-ÖV-Kombiniererin Paula kommen aus Altersgründen oder wegen körperlicher Beeinträchtigungen manche Verkehrsmittel nur noch eingeschränkt in Frage. Der Aspekt der Materialität, sowohl bezogen auf Verkehrsmittel als auch auf die Körperlichkeit der Person, wird damit deutlich. Nutzbar scheinen nur die Verkehrsmittel, die ein bestimmtes Maß an Sicherheit, Zugänglichkeit und Komfort bieten, was aus Paulas Sicht häufig nur der Pkw, teilweise der ÖV und eingeschränkt das Fahrrad sind. Anders als bei der ÖV-Nutzerin spielt Zeit- oder Geldersparnis eher keine Rolle.

Im nahen Umfeld scheint die Nutzung des eigenen Fahrzeuges die komfortabelste und routinierteste Lösung zu sein: „Also das Auto nehme ich sehr häufig, so als Anfahrt, um die Wege, die dann beschwerlich für mich sind, in dem Sinne, dass ich kein Fahrrad nehmen kann, oder dass zu Fuß zu weit ist " $(\mathrm{P} 1)$. Für weitere Fahrten in der Stadt ist die Kombination des Pkws mit dem ÖV für sie die beste Option, da sie aufgrund ihrer Erfahrung den als herausfordernd wahrgenommenen Innenstadtverkehr und die Parkplatzsuche vermeiden möchte. Die Kombination Pkw-ÖV ermöglicht das Wohnen am Stadtrand sowie die Partizipation am urbanen Leben in der Innenstadt. Die Interviewten schätzen diese als selbstbestimmt wahrgenommene Mobilität sehr: „So lange es geht behalt ich es [Auto], weil es mir diese Beweglichkeit gibt, es doch ein gutes Mittel ist im Alter lebendig zu bleiben und dieses Lebendigbleiben bringt ja auch Gesundheit mit sich und Wachheit und auch die Neugierde“ (P1). „Das mag ich sehr gerne und ich hoffe, dass ich noch länger mobil bleibe und mir das erhalten kann" (P3).

Bei der Beschreibung eines typischen Weges von Paula (vgl. Abb. 2) - die Fahrt mit dem Pkw von zuhause zur SBahnstation und mit der S-Bahn in die Innenstadt - wird klar, dass diese Praxis zu ihrer Routine geworden ist und die Situation an Ein- und Umstiegspunkten ihr wohl bekannt ist. Meist werden daher die gleichen Orte aufgesucht. Die Routine sowie ihr methodisches Wissen tragen zum Sicherheitsund Komfortempfinden Paulas bei, weil spontanes und flexibles Agieren an unbekannten Orten (z.B. ÖV-Stationen) dann nicht notwendig ist.

\section{Steffen (Intermodaler Fahrrad-Kombinierer)}

Der intermodale Fahrrad-Kombinierer Steffen nutzt bevorzugt das Fahrrad sowie den ÖV oder Carsharing, häufig in Kombination, um an sein Ziel zu gelangen. Bei unbekannten Strecken plant er, oft mit Hilfe von MobilitätsApps, welche Verkehrsmittel genutzt und kombiniert werden können. Dabei bedient er sich routiniert verschiedener Apps, die auf das jeweilige Verkehrsmittel abgestimmt sind. Dementsprechend verfügt er auch häufig über ÖV-Karten und Sharing-Mitgliedschaften. Hier kommt, analog zur PkwÖV-Kombiniererin Paula, die Verknüpfung von Materialität, (methodischem) Wissen sowie Routinen in seiner Mobilitätspraktik zum Ausdruck.

Steffen möchte so schnell wie möglich an sein Ziel kommen, wobei ihm die flexible Auswahl verschiedener Mobilitätsangebote hilft, dies umzusetzen. Häufig wohnt er urban, arbeitet Vollzeit und hat eine Familie, wodurch tagtäglich verschiedene Wege und Bedürfnisse zeiteffizient gemanagt werden müssen. Das Fahrrad ist aus Steffens Sicht dafür das schnellste und flexibelste Fortbewegungsmittel. Steffen verfügt zudem über gute Ortskenntnisse, was einen weiteren Effizienzfaktor für ihn darstellt. 
Das Auto ist dabei für Steffen keine attraktive Alternative, da Autofahren als unnötig und unpraktisch empfunden wird: „Ich finde Autofahren anstrengend, gerade besonders in der Stadt. Ich finde das echt nervig. [...] inzwischen ist es so voll und ich finde es wirklich stressig. Und dann immer diese Parkplatzsucherei, schrecklich" (R1). Ein Auto nutzt Steffen nur, ,wenn ich etwas zu transportieren habe, was halt größer ist “ (R3). In diesen Fällen nutzt er aufgrund deren Flexibilität häufig Carsharing-Angebote.

Generell trifft Steffen seine Wahl eher zweckorientiert und spontan, aber auch präferenzorientierte Gründe, also sein motivational-emotionales Wissen, beeinflussen seine Mobilitätspraktik. Beispielsweise ist es ihm wichtig, beim Fahrradfahren an der frischen Luft sein zu können. Zudem denkt Steffen über das Gemeinwohl und den unterschiedlichen Nutzen, den Mobilitätsoptionen für ihn und die anderen Verkehrsteilnehmer haben, nach.

\section{Situationsabhängige multimodale Nutzerin (Silvia)}

Die situationsabhängig multimodale Nutzerin Silvia wählt pragmatisch und situationsbedingt ein Verkehrsmittel. Zeitersparnis spielt eine große Rolle. Anders als beispielsweise der Allzweck-Pkw-Nutzer hat sie aber keine prinzipielle Präferenz für ein bestimmtes Verkehrsmittel und keine besonders ausgeprägte Routine. Stattdessen ist ihrem Unterwegssein ein anspruchsvoller Abwägungsprozess von Vor- und Nachteilen verschiedener Verkehrsmittel vorgeschaltet, bei dem - basierend auf ihren Erfahrungen und ihrem Wissen - mal pragmatische und mal emotionale Aspekte Einfluss zeigen. Zur Arbeit fährt sie beispielsweise mit dem ÖV, weil das die zeiteffizienteste Lösung ist. Sobald der Weg mit dem Pkw leichter und flexibler zu absolvieren ist, wird auch dieser genutzt: „Also Freunde besuchen auch wenn Sie nicht unbedingt öffentlich gut erreichbar sind oder wenn man abends flexibler sein will“ (S2). Silvia schätzt aber auch den Komfort eines Pkws, zum Beispiel bei schlechtem Wetter: „Wenn es regnet natürlich. Es ist gut, wenn man so ein Ding [Pkw] hat, finde ich wirklich luxuriös und nicht durch den Regen laufen zu U-Bahn und S-Bahn" (S4). Gute Ortskenntnisse sowie ein breites Wissen über unterschiedliche Mobilitätsangebote sind die Grundlage für die situationsangepasste Entscheidung für ein Verkehrsmittel. Teil dessen sind auch Mobilitäts-Apps, die helfen, den optimalen Weg von A nach B zu finden: „Also da würde ich mich [...] erstmal über die App erkundigen wie viel Zeit ich brauche und würde dann vergleichen, wie viel ich mit dem Auto brauche und würde dann nach der Zeit entscheiden, wie ich am schnellsten eben zurück komme“ (S2).

Grundsätzlich wird das Auto eher als verzichtbarer Luxus und nicht als Notwendigkeit wahrgenommen. ,Mit dem Auto ab und zu, muss aber nicht sein, wenn ich rausfahre aus der Stadt schon und wenn ich was Größeres transportieren muss auch, aber sonst steht das meistens rum [...] Im Moment ist es eine etwas luxuriöse Situation, weil im Grunde brauchen wir es eigentlich nicht" (S4). Gleichzeitig erfordert der Lebensalltag Silvias zwischen ihrer Arbeit, ihrem Sozialleben und ihrer Familie ein komplexes Management von Mobilitätsbedürfnissen. Um diese - idealerweise effizient - bewältigen zu können, fällt Silvias Wahl dennoch gelegentlich auf den Pkw: „Früher als die Kinder noch zu Hause waren, brauchte man natürlich mehr, dann war ich auch öfter mit dem Auto unterwegs [...] und dann kann man auch gleich verschiedene Sache in einem Ritt erledigen " (S2).

\section{Diskussion der Mobilitätstypen und Anknüpfungspunkte für die Praxis}

Die Ergebnisse der Mixed-Methods-Studie zeigen, dass es in der Stadt heutzutage eine ganze Bandbreite an Mobilitätstypen mit unterschiedlichen Praktiken, aber auch unterschiedlichen, den Praktiken zugrunde liegenden Logiken gibt. Sowohl die quantitative als auch die qualitative Analyse und Darstellung der unterschiedlichen Mobilitätspraktiken und vor allem Nutzungspraktiken des Pkws haben gezeigt, dass es nicht den einen ,Pkw-Nutzer“ gibt, wie er in einigen Mobilitätstypologien zum Beispiel neben dem Typ „FahrradFahrer" präsentiert wird (z.B. Hunecke und Haustein, 2007: „Pkw-Individualisten“ vs. „Radfans“ etc.). Stattdessen wird in diesem Beitrag die Vielfalt intermodaler und multimodaler Verhaltensweisen bei der Pkw-Nutzung deutlich. Die Ergebnisse bestätigen existierende Studien in dem Punkt, dass die Pkw-Nutzung bei einigen Nutzertypen durch emotionale, symbolische Gründe (Schlag und Schade, 2007; Steg, 2005) oder eine feste Nutzungsroutine motiviert ist (Tertoolen et al., 1998; Gärling und Axhausen, 2003). Gleichzeitig sind bei anderen Nutzertypen pragmatische Faktoren verantwortlich für die Pkw-Nutzung (Alteneder und Risser, 1995; Steg et al., 2001). Die letztendlich vollzogene Praktik - die PkwNutzung - ist dabei womöglich dieselbe. Die dahinterliegenden Logiken unterscheiden sich jedoch stark. Materielle Parameter wie Zeit und Geld, die in der Literatur meist als die wichtigsten zur Erklärung der Verkehrsmittelwahl angeben werden, sind nicht für jeden Typ (gleich) bedeutend. So sind für den Allzweck-Pkw-Nutzer emotional-motivationale Aspekte, wie die Qualität der Zeit beim Fahren, wichtig. Das Fahren wird häufig als Genuss oder me-time empfunden und wertgeschätzt. Dagegen sind für die situationsbedingte multimodale Nutzerin und die ÖV-Nutzerin Zeiteffizienz bzw. ersparnis wichtig, und die Aufenthaltsqualität im Verkehrsmittel spielt eine untergeordnete Rolle. Es gilt in der Praxis Lösungen zu finden, die diese unterschiedlichen Logiken der Zeitnutzung und -wahrnehmung berücksichtigen.

Für diejenigen, für die das Auto ein Ort der Entspannung und me-time bedeutet, könnten Luxus-Varianten von 
on-demand „Ridepooling“3 ${ }^{* 3}$ als iÖV (individualisierter öffentlicher Verkehr) eine Alternative sein. Aktuelle Beispiele von On-demand-Mobilitätsangeboten zeigen erste Ideen, wie diese Privatheit im iÖV beispielsweise durch mehr Platz, Distanz zu Mitfahrenden oder Sichtschutz gewährleistet werden könnte.

In vielen Interviewpassagen kommt zum Ausdruck, dass die Pkw-Nutzung bei der situationsabhängigen multimodalen Nutzerin (Silvia) und auch bei der ÖV-Nutzerin (Olga) meist aus einem Pragmatismus heraus zu erklären ist. Auch der intermodale Fahrrad-Kombinierer (Steffen) und die intermodale Pkw-und-ÖV-Kombiniererin (Paula) nutzen das Auto häufig aus organisatorischen Gründen und zur Bewältigung von familiären Sozialaktivitäten, was auch Dowling und Maalsen (2020) in ihrer Studie in Bezug auf Familienmobilität beschreiben. Auch Manderscheid (2019) vermutete bereits eine starke Kopplung von Familienmobilität und Auto-Praktiken mit solchen Logiken. Der private Pkw erfüllt damit häufig die Transport-, cocooning- und Bequemlichkeits-Bedürfnisse einer Familie. Kent und Dowling (2016) mutmaßen, dass die Idee von „Mobility-as-aService“ (MaaS) ${ }^{4}$ ein Konzept sein könnte, das der Flexibilität, die ein Auto bietet, nahekommt und somit eine Alternative zum privaten Pkw sein könnte. Bestimmte Anforderungen bzw. Situationen könnten dadurch adressiert werden. Vor dem Hintergrund der Ergebnisse dieser Studie ist jedoch nur schwer vorstellbar, dass das Familien-Auto, das eine Reihe von Aufgaben erfüllt, dadurch gänzlich ersetzt werden kann. Auch werden die digitale Informationsbeschaffung und Bedienung der neuen Angebote von vielen der Befragten zunächst als unzufriedenstellend wahrgenommen. Das Herunterladen einer weiteren App, die Anmeldung etc. werden als Barriere gesehen. Eine reduzierte Komplexität digitaler Anwendungen würde in vielen Fällen zu einer höheren Nutzerakzeptanz führen.

Es ist festzuhalten, dass das Auto nicht für alle AutonutzerInnen, mit Alteneder und Risser (1995) sprechend, ,ein Symbol für tief verwurzelte ideologische und psychologische Projektion ist und rationale Argumente eher im Hintergrund stehen“ (1995: 80). Während für den AllzweckPkw-Nutzer (Peter) das Wohlbefinden, die Unabhängigkeit und andere interpretativ-symbolische und emotionalmotivationale Aspekte sowie die etablierte Routine des Autofahrens durchaus wichtig sind, sind für Silvia materielle Anforderungen, wie zum Beispiel der Transport von Gütern oder die Beförderung von Personen, stärkere Gründe für die Praxis der Autonutzung. Individualistische Einstellungen, wie ein Gefühl der Unabhängigkeit, das oft dem Au-

\footnotetext{
${ }^{3}$ Beim Ridepooling werden mehrere Personen mit ähnlichem Ziel anhand eines IT-Algorithmus gebündelt (Kloth und Mehler, 2018: 37f).

${ }^{4}$ Mobility-as-a-Service (MaaS) kombiniert öffentliche und private Verkehrsangebote unterschiedlicher Anbieter mittels einheitlichem Buchungsportal (Bitkom, 2018).
}

tofahren zugeschrieben wird (te Brömmelstroet et al., 2017), konnten bestätigt werden. Diese lassen sich einerseits einer unmittelbaren emotionalen Motivation und andererseits einem interpretativen Verstehen sozialer Symboliken von Freiheit und Unabhängigkeit zuschreiben, bzw. deren Materialisierung im Objekt „Auto“. Ähnliches gilt aber auch für den Fahrradfahrer Steffen bezogen auf sein Fahrrad, das für ihn Flexibilität und Freiheit symbolisiert. Für diejenigen, die hauptsächlich ein Verkehrsmittel (Auto oder Fahrrad) nutzen, treiben symbolisch-interpretative Aspekte häufig eine eher individuale Organisation ihrer Mobilität.

Gemein haben die Allzweck-Pkw-Nutzer und intermodalen Fahrrad-Kombinierer die Präferenz, Nutzungsroutine sowie Passion für das jeweilige Verkehrsmittel. Die Analyse der Logiken hinter diesen Mobilitätspraktiken zeigt, dass bei den Individualverkehrsmitteln eine Entscheidung für diese gefällt wird, wohingegen die Nutzung öffentlicher Verkehrsmittel eher das Ergebnis der Entscheidung gegen andere ist (Pkw ist zu teuer, Fahrrad ist zu wetterabhängig etc.). Das Herz der ÖV-Nutzerin oder der Pkw-undÖV-Kombiniererin schlägt also nicht für die gewählte Option. Emotional-motivationale und symbolisch-interpretative Aspekte sind für sie weniger einflussreich. Ausschlaggebender sind die materiellen Anforderungen ihrer Mobilität, die ein pragmatischeres und weniger stark routinisiertes Abwägen erfordern.

Für Paula, die intermodale Pkw-und-ÖV-Kombiniererin, sind weder äußere materielle Beschränkungen wie Zeit und Geld noch interpretativ-symbolische, affektive Gründe ein entscheidender Faktor bei ihrer Praxis. Paula hat viel Zeit als Rentnerin und ihr ist bewusst, dass die Haltung eines eigenen Pkws kostenintensiv ist. Dennoch ist ihr der Besitz eines eigenen Autos sehr wichtig. Entscheidender Faktor ist für sie die gesellschaftliche Teilhabe - wie zum Beispiel der Theaterbesuch in der Innenstadt -, der ihr durch den Besitz eines eigenen Autos auch im hohen Alter möglich ist (analog das „Motiv der Zusammenkunft“ und der ,,routinisierte Rhythmus sozialer Ereignisse“ bei Wilde, 2014: 151-152). Der Mobilitätstyp Paula macht deutlich, dass auch Angebote für ältere, möglicherweise in ihrer Mobilität eingeschränkte und weniger digital- und technikaffine Bevölkerungsgruppen sowie in weniger urbanen Stadtquartieren geschaffen werden müssen.

Vergleicht man die Ergebnisse dieser Studie mit denen vergangener Typologie-Studien (etwa Götz et al.,1998; Prillwitz und Barr, 2011), dann lassen sich - trotz der zeitlichen Distanz und einer anderen theoretischen Brille - Parallelen feststellen. Ein auffälliger Unterschied zu diesen Typologien ist das Aufkommen von situativen, multimodalen Nutzungspraktiken von Pkws. Das Aufkommen von alternativen Mobilitätsangeboten und die höhere Planungsflexibilität durch digitale Angebote sind dafür wahrscheinliche Auslöser. Generell bestätigt sich aber die schon damals gemachte Beobachtung einer tiefgreifenden Verwurzelung der Automobilität auf der interpretativ-symbolischen Ebene sowie auf der 
Ebene materieller Anforderungen, insbesondere bei Familien.

Die Erkenntnisse dieser Studie machen deutlich, dass bei der Entwicklung von Maßnahmen oder neuen Mobilitätsangeboten, die dazu führen sollen, den Pkw-Besitz und dessen Nutzung zu reduzieren, die reine Betrachtung des Mobilitätsverhaltens nicht ausreicht. Alternativlösungen sollten ausgehend von den Mobilitätslogiken und insbesondere mit der Auto-Nutzung verbundenen Logiken entwickelt werden, um diese längerfristig und zielgruppenspezifisch mit entsprechenden bedarfsgerechten Angeboten zu adressieren. Was offen bleibt ist die Frage, ob Maßnahmen und neue Angebote, die auf Verhaltensänderungen wie die Pkw-Abschaffung abzielen, Personen (beispielsweise den Allzweck-Pkw-Nutzer Peter) zum Umdenken bewegen. Zurecht gibt es Stimmen, die davon ausgehen, dass dadurch bisher wenig signifikante Veränderungen angestoßen worden sind (Manderscheid, 2019). So wird einE NutzerIn, der/die beispielsweise bereits eine grundsätzliche Entscheidung für das Auto getroffen hat, schwer davon zu überzeugen sein, spontan und situativ ein alternatives Mobilitätsangebot zu nutzen. Zukünftig könnte jedoch durch demographische und gesellschaftliche Entwicklungen die Gruppe der situationsbedingten multimodalen NutzerInnen zulasten der AllzweckPkw-NutzerInnen stärker an Bedeutung gewinnen. Für eine abschließende Bewertung dessen bedarf es empirischer Langzeitstudien, die Verhaltens- und Praxisveränderungen aufgrund neuer Angebote oder Maßnahmen in den Blick nehmen.

\section{Fazit \& Ausblick}

Die vorliegende Studie zeigt den Mehrwert des MixedMethods-Ansatzes für die Bildung und das tiefgehende Verstehen von Mobilitätstypen, deren Mobilitätspraktiken und Logiken. Die Identifizierung von Mobilitätstypen auf der Grundlage von quantitativen Daten reduziert die Komplexität und ermöglicht eine gezielte Untersuchung der der Praktik zu Grunde liegenden Mechanismen. Die Einbeziehung inter-, multi- und unimodaler Verkehrsmittelnutzungen in die Identifizierung von Typen scheint dabei die Realität treffender abzubilden als eine Strukturierung nach Hauptverkehrsmitteln. Darüber hinaus liefern die aus den qualitativen Interviews gewonnenen Erkenntnisse zu den Praktiken unterschiedlicher Mobilitätstypen sowie die der Autonutzung zu Grunde liegenden Logiken wertvolle Informationen für die Entwicklung nutzergruppenspezifischer Maßnahmen und bedarfsgerechter Mobilitätskonzepte. Durch den Blick auf die individuelle Lebenswelt konnten Mobilitätspraktiken und deren Logiken mit deren subjektiver Rationalisierung ins Verhältnis gesetzt werden, woraus sich Aussagen über die erwartbare Effektivität von Ansätzen der Planungspraxis besser einschätzen ließen.
Für zukünftige Forschungsarbeiten wäre die Übertragung des vorgestellten Ansatzes in einen anderen räumlichen Kontext sehr interessant. Auch wenn davon auszugehen ist, dass ähnliche Mobilitätspraktiken und Logiken in anderen Großstädten festzustellen sind, stehen die vorgestellten Ergebnisse für die Stadt Berlin. Da jede Stadt ihre eigenen Charakteristika und einen spezifischen Kontext hat, gilt es, die Verallgemeinerung der Ergebnisse mit Vorsicht vorzunehmen.

Die Studie zeigt die mögliche Vielfalt von (automobilen) Mobilitätspraktiken in der Stadt, die keine Abfolge von isolierten, rationalen Entscheidungen sind, sondern komplexe, soziale Praktiken darstellen, die nur in Zusammenhang mit ihren Materialitäten, dem mit ihnen verknüpften Wissen und ihren Routinen zu verstehen sind. Einzelne Praktiken, wie zum Beispiel die Pkw-Nutzung, können darüber hinaus verschiedene Akteure mit unterschiedlichen Logiken anziehen. Es wird deshalb klar, dass diese Vielfalt an Logiken auch für die Entwicklung von neuen Mobilitätskonzepten wichtig ist und mit passenden Methoden adressiert werden sollte. Es sind weitere Forschungsstudien erforderlich, die sich auf diese weichen, nur schwer quantitativ zu messenden Faktoren konzentrieren. Das Konzept der Logiken von Mobilitätspraktiken könnte ein weiterer Schritt dabei sein, die bestehenden Ansätze einer Operationalisierung der Praxistheorien für die Mobilitätsforschung (v. a. von Wilde) weiterzuentwickeln und damit einen Analyserahmen sowohl für die Anforderungen der Erforschung von Lebenspraktiken zu schaffen als auch den Anforderungen der Planungspraxis gerecht zu werden.

Datenverfüg barkeit. Die für diesen Artikel genutzten Datensätze (quantitative Befragungsdaten und Interviewtranskripte) sind aufgrund der Datenschutzbestimmungen der Erhebungen nicht öffentlich zugänglich.

Autorlnnenmitwirkung. LG und RO haben gemeinsam den Artikel konzipiert, die Datenerhebung und -analysen durchgeführt sowie den Text verfasst und überarbeitet. LG entwickelte den theoretischen Bezug des Themas. Die Hauptverantwortung für die Verfassung des Artikels sowie die Projektakquise lag ebenfalls bei LG.

Interessenkonflikt. Die Autorinnen erklären, dass kein Interessenkonflikt besteht.

Danksagung. Wir bedanken uns bei den GutachterInnen sowie bei unseren KollegInnen Jakob Schmitz, Heike Marquart und Kerstin Stark für wertvolle Kommentare zum Manuskript.

Finanzierung. Die Forschungsarbeiten waren Teil des Projekts "Urbane Mobilität", institutionell gefördert durch das Deutsche Zentrum für Luft- und Raumfahrt (DLR). 
Begutachtung. This paper was edited by Nadine Marquardt and reviewed by two anonymous referees.

\section{Literatur}

Alteneder, W. und Risser, R.: Soziologie der Verkehrsmittelwahl. Motive und Bedürfnisse im Zusammenhang mit der Verkehrsmittelwahl, Zeitschrift für Verkehrssicherheit, 41, 77-83, 1995.

Anable, J.: 'Complacent Car Addicts' or “Aspiring Environmentalists"? Identifying Travel Behaviour Segments Using Attitude Theory, Transport Policy, 12, 65-78, 2005.

Bartz, F. M.: Mobilitätsbedürfnisse und ihre Satisfaktoren. Die Analyse von Mobilitätstypen im Rahmen eines internationalen Segmentierungsmodells, Dissertation, Humanwissenschaftliche Fakultät, Universität zu Köln, Köln, 274 pp., 2015.

Behnken, I. und Zinnecker, J.: Methoden der empirischen erziehungswissenschaftlichen Forschung. Narrative Landkarten. Ein Verfahren zur Rekonstruktion aktueller und biografisch erinnerter Lebensräume, Zeitschrift Enzyklopädie Erziehungswissenschaft Online, 1-25, https://doi.org/10.3262/EEO07100128, 2010.

Bitkom: White Paper MaaS - Mobility-as-a-Service Chancen für Mobility-as-a-Service-Geschäftsmodelle, abrufbar unter: https://bitkom.org/sites/default/files/file/ import/181016-White-Paper-MaaS.pdf (zuletzt aufgerufen: 1 April 2021), 2018.

BMVI: Verkehr in Zahlen 2017/2018, Verkehr in Zahlen, Bundesministerium für Verkehr und digitale Infrastruktur, Hamburg, Germany, 2017.

Bolte, K. M.: Typen alltäglicher Lebensführung, in: Lebensführung und Gesellschaft. Beiträge zu Konzept und Emperie alltäglicher Lebensführung, Herausgeber: Kudera, W. und Voß, G. G., Leske + Budrich, Opladen, 133-146, 2000.

Chapman, C. N. und Milham, R. P.: The Personas' New Clothes: Methodological and Practical Arguments against a Popular Method, Proceedings of the Human Factors and Ergonomics Society Annual Meeting, 50, 634-636, https://doi.org/10.1177/154193120605000503, 2006.

Charmaz, K.: Constructing Grounded Theory. A Practical Guide Through Qualitative Analysis 2ed., Sage Publications, London, 2014.

Chlond, B.: Mulitmodalität und Intermodalität, in: Nicht weniger unterwegs, sondern intelligenter? Neue Mobilitätskonzepte, edited by: Beckmann, K. J. und Klein-Hitpaß, A., Edition Difu, 11, Deutsches Institut für Urbanistik Difu, Berlin, 271-293, 2013.

Collier, J. und Collier, M.: Visual anthropology. Photography as a research method, University of New Mexico Press, Albuquerque, 1986.

Cooper, A.: Inmates Are Running the Asylum: Why High-Tech Products Drive Us Crazy and How to Restore the Sanity, Sams, ISBN 0672326140, 2004.

Dangschat, J. S.: Wie bewegen sich die (Im-)Mobilen? Ein Beitrag zur Weiterentwicklung der Mobilitätsgenese, in: Verkehr und Mobilität zwischen Alltagspraxis und Planungstheorie. Ökologische und soziale Perspektiven, Herausgeber: Wilde, M., Gather, M., Neiberger, C., und Scheiner, J., Studien zur Mobilitäts- und Verkehrsforschung, Springer VS, Wiesbaden, Germany, 25-51, 2017.
Dangschat, J. S. und Segert, A.: Nachhaltige Alltagsmobilität - soziale Ungleichheiten und Milieus, Österreichische Zeitschrift für Soziologie, 36, 55-73, 2011.

De Vos, J., Mokhtarian, P. L., Schwanen, T., Van Acker, V., und Witlox, F.: Travel mode choice and travel satisfaction: bridging the gap between decision utility and experienced utility, Transportation, 43, 771-796, 10.1007/s11116-015-9619-9, 2016.

Docherty, I., Marsden, G., und Anable, J.: The governance of smart mobility, Transport. Res. A, 115, 114-125, 10.1016/j.tra.2017.09.012, 2017.

Dowling, R. und Maalsen, S.: Familial mobilities beyond the private car: electric bikes and car sharing in Sydney, Australia, Applied Mobilities, 5, 53-67, https://doi.org/10.1080/23800127.2019.1571658, 2020.

Gärling, T. und Axhausen, K. W.: Introduction: Habitual Travel Choice, Transportation, 30, 1-11, 2003.

Giddens, A.: The constitution of society: Outline of the theory of structuration, Polity, Cambridge, University of California Press, Berkeley and Los Angeles, 1984.

Glaser, B. und Strauss, A.: The Discovery of Grounded Theory, IL, Aldine, Routledge, London, New York, 1967.

Götz, K., Jahn, T., und Schultz, I.: Mobilitätsstile - ein sozialökologischer Untersuchungsansatz, Institut für sozial-ökologische Forschung, Frankfurt am Main, 1998.

Hannam, K., Sheller, M., und Urry, J.: Editorial: Mobilities, Immobilities and Moorings, Mobilities, 1, 1-22, https://doi.org/10.1080/17450100500489189, 2006.

Harper, D.: Fotografien als sozialwissenschaftliche Daten, in: Qualitative Forschung. Ein Handbuch, Herausgeber: Flick, U., Kardorff, E., und von Steinke, I., Rowohlt, Reinbek bei Hamburg, 402-416, 2009.

Haustein, S. und Nielson, T. A. S.: European Mobility Cultures: A Survey-Based Cluster Analysis Across 28 European Countries, J. Transp. Geogr., 54, 173-180, 2016.

Hunecke, M.: Ansätze zur Segmentierung von NutzerInnengruppen, in: Mobilitätsverhalten verstehen und verändern. Studien zur Mobilitäts- und Verkehrsforschung, Herausgeber: Hunecke, M., Springer VS, Wiesbaden, 47-74, 2015.

Hunecke, M. und Haustein, S.: Einstellungsbasierte Mobilitätstypen: Eine integrierte Anwendung von multivariaten und inhaltsanalytischen Methoden der empirischen Sozialforschung zur Identifikation von Zielgruppen für eine nachhaltige Mobilität, Umweltpsychologie, 11, 38-68, 2007.

Jensen, O. B.: Flows of Meaning, Cultures of Movements - Urban Mobility as Meaningful Everyday Life Practice, Mobilities, 4, 139-158, https://doi.org/10.1080/17450100802658002, 2009.

Johnson, R. und Onwuegbuzie, A. J.: Mixed Methods Research: A Research Paradigm Whose Time Has Come, Educ. Res., 33, 14, https://doi.org/10.3102/0013189X033007014, 2004.

Johnson, R., Onwuegbuzie, A. J., und Turner, L. A.: Toward a Definition of Mixed Methods Research, J. Mix. Method. Res., 1, 112-133, https://doi.org/10.1177/1558689806298224, 2007.

Kent, J. L. und Dowling, R.: The Future of Paratransit and DRT: Introducing Cars on Demand, in: Paratransit: Shaping the Flexible Transport Future, Herausgeber: Mulley, C. und Nelson, J. D., Emerald Group Publishing Limited, Bingley, 391-412, 2016.

Kloth, H. und Mehler, S.: Nachfragegesteuerte Verkehre oder OnDemand-Ridepooling?, Der Nahverkehr, Vol. 36, 6/2018, 36-39, ALBA-FACHVERLAG, ISSN 0722-8287, 2018. 
Kuckartz, U.: Qualitative Inhaltsanalyse. Methoden, Praxis, Computerunterstützung, 3 ed., Beltz Juventa Verlag GmbH, Weinheim, 240 pp., 2016.

Lanzendorf, M. und Hebsaker, J.: Mobilität 2.0 - Eine Systematisierung und sozial-räumliche Charakterisierung neuer Mobilitätsdienstleistungen, in: Verkehr und Mobilität zwischen Alltagspraxis und Planungstheorie, Herausgeber: Wilde, M., Gather, M., Neiberger, C., und Scheiner, J., Springer VS, Wiesbaden, 135151,2017

Le Bris, J.: Die individuelle Mobilitätspraxis und Mobilitätskarrieren von Pedelec-Besitzern: Adoption und Appropriation von Elektrofahrrädern, Dr. phil., MathematischNaturwissenschaftlichen Fakultät, Eberhard Karls Universität Tübingen, Tübingen, 2015.

Manderscheid, K.: Auto-logische Koppelung: eine quantitativpraxistheoretische Perspektive auf Mobilität, Schweizerische Zeitschrift für Soziologie, Revue suisse de sociologie, 45, 161183, 10.2478/sjs-2019-0008, 2019.

Oostendorp, R. und Gebhardt, L.: Combining Means of Transport as a Users' Strategy to Optimize Traveling in an Urban Context: Empirical Results on Intermodal Travel Behavior from a Survey in Berlin, J. Transp. Geogr., 71, 72-83, 2018.

Oostendorp, R., Nieland, S., und Gebhardt, L.: Developing a User Typology Considering Unimodal and Intermodal Mobility Behavior: A Cluster Analysis Approach Using Survey Data, Eur. Transp. Res. Rev., 11, 33, https://doi.org/10.1186/s12544-0190369-1, 2019.

Prillwitz, J. und Barr, S.: Moving Towards Sustainibility? Mobility Styles, Attitudes and Individual Travel Behaviour, J. Transp. Geogr., 19, 1590-1600, 2011.

Reckwitz, A.: Grundelemente einer Theorie sozialer Praktiken: Eine sozialtheoretische Perspektive, Zeitschrift für Soziologie, 32, 282-301, 2003.

Rohrauer, B.: Die Erweiterung der Nadelmethode und das Potential aktueller kartenbasierter Technologien für die sozialräumliche Methodenentwicklung, soziales_kapital, 12, abrufbar unter: https://soziales-kapital.at/index.php/sozialeskapital/ article/view/340/584 (zuletzt aufgerufen: 28 January 2020.), 2014

Schatzki, T. R.: Social Practices. A Wittgensteinian Approach to Human Activity and the Social, Cambridge University Press, Cambridge, 1996.

Schatzki, T. R.: A Primer on Practices, in: Practice-Based Education. Practice, Education, Work and Society, SensePublishers, Rotterdam, 2012.

Scheiner, J.: Verkehrsgeneseforschung, in: Handbuch Verkehrspolitik, Herausgeber: Schöller, O., Canzler, W., und Knie, A., VS Verlag für Sozialwissenschaften, Wiesbaden, 687-709, 2007.

Scheiner, J.: Review: Mobilität und Alltag. Einblicke in die Mobilitätspraxis älterer Menschen auf dem Lande by Mathias Wilde, Erdkunde, 68, 148-151, 2014.

Schlag, B. und Schade, J.: Psychologie des Mobilitätsverhaltens, Aus Politik und Zeitgeschichte, 29, 27-32, 2007.

Schopf, J. M.: Mobilität \& Verkehr - Begriffe im Wandel, in: Verkehr und Mobilität, Herausgeber: Knoflacher, H., Wissenschaft \& Umwelt INTERDISZIPLINÄR, Wien, 3-11, abrufbar unter: https://docplayer.org/15071931-Wissenschaftumwelt-interdisziplinaer-3-verkehr-, und-mobilitaet.html (zuletzt aufgerufen: 1 April 2021), 2001.
Schwanen, T., Banister, D., und Anable, J.: Rethinking Habits and their Role in Behaviour Change: The Case of Low-carbon Mobility, J. Transp. Geogr., 24, 522-532, 2012.

Segert, A.: Mobilitätsorientierungen - eigenständiger Faktor für die Entwicklung nachhaltiger Mobilität in ländlichen Räumen, Ländlicher Raum, 1-17, abrufbar unter: https://www. bmlrt.gv.at/dam/jcr:e35cdd20-6249-4dba-9682-9ecc126a659a/ AstridSegert-Mobilit\%C3\%A4tsorientierungen-eigenst\%C3\% A4ndigerFaktorf\%C3\%BCrdieEntwicklungnachhaltigerMobilit\% C3\%A4t.pdf (zuletzt aufgerufen: 2 April 2021), 2009.

Shove, E., Pantzar, M., und Watson, M.: The Dynamics of Social Practice. Everyday Life and How It Changes, Sage, Los Angeles, London, New Delhi, Singapore, Washington DC, 2012.

Steg, L.: Car Use: Lust and Must. Instrumental, Symbolic and Affective Motives for Car Use, Transportation Research Part A: Policy and Practice, 39, 147-162, 2005.

Steg, L., Vlek, C., und Slootegraf, G.: Instrumental-Reasoned and Symbolic-Affective Motives for Using a Motor Car, Transportation Research Part F: Traffic Psychology and Behaviour, 4, 151169, 2001.

te Brömmelstroet, M., Nikolaeva, A., Glaser, M., Nicolaisen, M. S., und Chan, C.: Travelling Together Alone and Alone Together: Mobility and Potential Exposure to Diversity, Applied Mobilities, 2, 1-15, https://doi.org/10.1080/23800127.2017.1283122 2017.

Tertoolen, G., van Kreveld, D., und Verstraten, B.: Psychological resistance against attempts to reduce private car use, Transportation Research Part A: Policy and Practice, 32, 171-181, https://doi.org/10.1016/S0965-8564(97)00006-2, 1998.

Urry, J.: Mobilities, Polity Press, Cambridge, 2007.

Venn, C.: Individuation, relationality, affect: rethinking the human in relation to the living, Body Soc., 16, 129-161, 2010.

WBGU: Welt im Wandel. Gesellschaftsvertrag für eine Große Transformation. Hauptgutachten, Wissenschaftlicher Beirat der Bundesregierung Globale Umweltveränderungen, Berlin, 2011.

Wilde, M.: Mobilität als soziale Praxis: ein handlungstheoretischer Blick auf Bewegung, in: Mobilitäten und Immobilitäten Menschen - Ideen - Dinge - Kulturen - Kapital, 1. Aufl. ed., Herausgeber: Scheiner, J., Blotevogel, H. H., Holz-Rau, C., und Schuster, N., Dortmunder Beiträge zur Raumplanung : Blaue Reihe, 142, Klartext, Essen, 35-48, 2013.

Wilde, M.: Mobilität und Alltag. Einblicke in die Mobilitätspraxis älterer Menschen auf dem Land, Studien zur Mobilitäs- und Verkehrsforschung, 25, Herausgeber: Gather, M., Kagermeier, A., Kesselring, S., Lanzendorf, M., Lenz, B., und Wilde, M., Springer VS, Wiesbaden, 190 pp., 2014.

Wilde, M. und Klinger, T.: Integrierte Mobilitäts-und Verkehrsforschung: zwischen Lebenspraxis und Planungspraxis, in: Verkehr und Mobilität zwischen Alltagspraxis und Planungstheorie, Springer, Wiesbaden, Deutschland, 5-23, 2017.

Wittwer, R.: Zwangsmobilität und Verkehrsmittelorientierung junger Erwachsener: eine Typologisierung, Institut für Verkehrsplanung und Straßenverkehr, Dresden, 2014.

Wörmer, S.: Berufliche Mobilität im Alltag. Praktiken und Formen alltäglicher Lebensführung. Schriften des Arbeitskreises Stadtzukünfte der Deutschen Gesellschaft für Geographie, Lit Verlag, Berlin, 2016. 